\title{
ASSESSMENT OF CONFIGURATION OF FOUR ISOMERIC $\alpha, \beta$-UNSATURATED SULFONES USING A LANTHANIDE SHIFT REAGENT
}

\author{
H. A. Selling* \\ Institute for Organic Chemistry TNO, $\uparrow$ Utrecht, The Netherlands
}

(Received in UK 10 March 1975; Accepted for publication 1 May 1975)

\begin{abstract}
Abotract-This report describes the application of a computer-aided Lanthanide Induced Shift (LIS)-analysis to discriminate between the four isomeric $\alpha, \beta$-unsaturated sulfones $1 \mathrm{a}-\mathrm{d}$. The effect of rotation around the $\mathrm{MeSO}_{2}-\mathrm{C}$ and MeS- $C$ bonds upon the agreement between experimental and calculated Eu(fod), induced shifts has been investigated.
\end{abstract}

IN A PREVIOUS paper the formation of the isomeric sulfones $(Z)^{-1}$ - methylsulfonyl - 2 - methylthio - 2 - (4 tolyl)ethene, 1a, and $(E)-1$ - methylsulfonyl - 1 methylthio - 2 - (4 - tolyl)ethene, $1 \mathrm{~b}$, from base-catalysed methanethiol addition to 1 - methylsulfonyl - 2 - (4 tolyl)ethyne and subsequent photoisomerization to their $E$ - and $Z$-isomers $1 c$ and $1 d$ respectively has been described.'<smiles>COS(C)=CC=Cc1ccc(C)cc1</smiles>

la<smiles>CO[S+](C)(C)C=Cc1ccc(C)cc1</smiles>

$\mathbf{1 b}$<smiles>CS/C(=C\S(C)(=O)=O)c1ccc(C)cc1</smiles>

Ic<smiles>COS(C)=C(C)SCc1ccc(C)cc1</smiles>

Id
The present report involves a study to the feasibility of discriminating between these isomers with the aid of Lanthanide Induced Shifts (LIS), in order to achieve a rapid structure assignment.

The LIS-technique has not been reported for sulfones so far, but its proven applicability to sulfoxides ${ }^{2-4}$ left little doubt as to its utility.

For pseudo-contact interactions and magnetically axially symmetric complexes the relationship between LIS and molecular geometry of the substrate ligand is represented by the well-known McConnell-Robertson equation:

$$
\Delta \nu_{i}=\mathrm{K} \cdot\left(3 \cos ^{2} \theta_{\mathrm{i}}-1\right) \cdot \mathrm{r}_{\mathrm{i}}^{-3}
$$

As a working hypothesis the above conditions are assumed to be satisfied. $11,12.18$

For structurally rigid systems the experimental LIS and those calculated by the McConnell-Robertson equation have successfully been matched by computer simulation. ${ }^{6-9}$ Recently a few reports on the application of LIS to the study of conformational equilibria in more fiexible systems have appeared..$^{10.17}$

fOrganization for Applied Scientific Research in The Netherlands.

\section{Theory}

The determination of the molecular geometry of the Lanthanide-substrate complex is best described by the model given in Fig. 1, applied on isomer 1a, using Eu(fod) as the LSR.

The origin of the coordinate system is defined by either of the sulfonyl oxygens which, in analogy with sulfoxides, $2-1$ is assumed to act as donor atom. The choice of the other $\mathrm{O}$ atom as the point of attachment to the lanthanide ion results in an equivalent model which is a mirror image of the former. The positive $y$-axis is defined by the direction of the $\mathrm{S}-\mathrm{C}$ bond.

The position of the Europium ion is determined by the three polar coordinates $R, \omega$ and $\varphi$, where $R$ represents the Eu-O internuclear distance, $\omega$ the angle between $\mathrm{Eu}-\mathrm{O}$ bond and positive $y$-axis and $\varphi$ the dihedral angle between the plane defined by Eu-O-y-axis and the $x, y$-plane, measured counter-clockwise.

A computer program evaluates for each spatial location of $\mathrm{Eu}$, defined by $\mathrm{R}, \omega$ and $\varphi$, the geometrical factor $\left(3 \cos ^{2} \theta_{i}-1\right) \cdot r_{1}^{-3}$ of the McConnell-Robertson equation, for all i protons. Subsequently the constant $K$ in eqn (1) is calculated according to the least squares procedure described by Lawton and Sylvestre, ${ }^{13}$ and the agreement between experimental and calculated LIS is expressed in terms of the normalized variance.' This procedure is repeated for different sets of $R, \omega$ and $\varphi$ using the direct search minimization method of Hooke and Jeeves. ${ }^{14}$ That

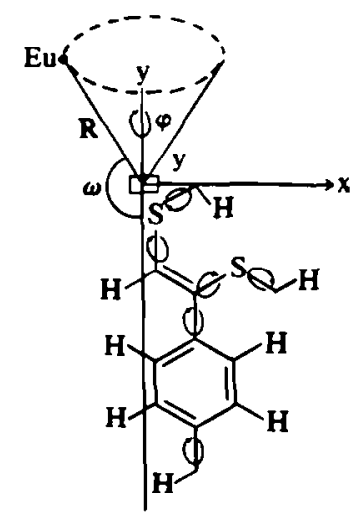

Fig. 1. Coordinate system for Eu(fod),-substrate complex, exemplified for 1a. R represents the Eu-O interatomic distance, $\omega$ the angle formed by Eu-donor atom bond and positive $y$-axis, $\varphi$ the azimuthal angle. The S-C bond is aligned parallel to the positive $y$-axis. One $\mathbf{C} \rightarrow \mathbf{O}$-atom of the sulfonyl group is placed in the origin, the other one is located on the positive $z$-axis. 


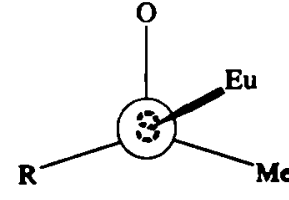

a

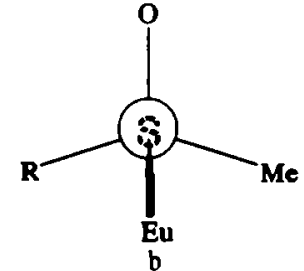

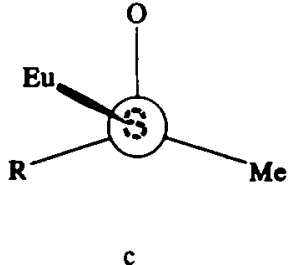

C

Fig. 2. Possible conformations around the $\mathrm{S}-\mathrm{O}$ bond. $\mathrm{R}$ : $\mathrm{CH}=\mathrm{C}(\mathrm{SMe}) \mathrm{C}_{6} \mathrm{H}_{4} \mathrm{Me}$ or $\mathrm{C}(\mathrm{SMe})=\mathrm{CHC}_{6} \mathrm{H}_{4} \mathrm{Me}$.

set of polar coordinates that corresponds to the lowest agreement factor is considered as the optimum Eulocation.

In analogy with Montaudo et al..$^{10}$ the $\mathrm{Eu}-\mathrm{O}$ distance was kept constant at 3.0 $\AA$ because of the reported low sensitivity of the agreement factor for variation of $R$ in the 2.5-3.5 $\AA$ range,, 10 and only $\omega$ and $\varphi$ were allowed to vary. The following further assumptions have been made:

(1) Free rotation occurs around the Eu-O interconnecting bond, thus canceling out deviations from axial symmetry of the magnetic susceptibility tensor. ${ }^{12}$

(2) Free rotation is also postulated for Me groups and for the Ph group, because of identical chemical shifts of the protons at opposite sites of the rotation axis. For these groups averaged proton coordinates have been utilized as input in the program.

(3) On the contrary free rotation is not very likely to occur around the $\mathrm{O}-\mathrm{S}$ bond, but rather preferred conformations will be taken in. Three possible conformations are depicted in Fig. 2, as has been suggested for sulfoxides. ${ }^{4}$ Conformation (b) can directly be ruled out due to steric hindrance of the $R$ and Me groups. The populations of the two remaining conformations will for the same reason ( $\mathrm{R}$ more bulky than $\mathrm{Me}$ ) be strongly biased towards rotamer (a). Evidence for this statement is obtained by a calculated rotamer population of only $10 \%$ for the rotamer which is syn to the bridge-head methyl

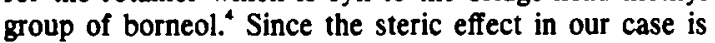
expected to be still more pronounced, it is assumed as an approximation that the complex exists only in one conformation around the $\mathrm{S}-\mathrm{O}$ bond.

(4) The conformations around the $\mathrm{MeSO}_{2}-\mathrm{C}$ bond are

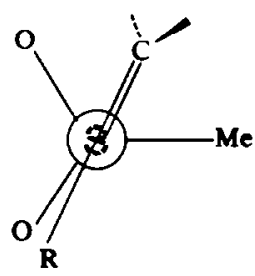

Fig. 3. Selected conformation about $\mathrm{SO}_{2}-\mathrm{C}$ axis. la and lc: $\mathrm{R}=\mathrm{H}$ : $1 \mathrm{~b}$ and $1 \mathrm{~d}: \mathrm{R}=\mathrm{SMe}$. also assumed to be restricted to only one, as shown in Fig. 3. This conformation has been chosen for all four isomers as a first approximation since from Dreiding models it is apparent that at least for 1a and 1c any other conformation experiences more steric hindrance.

(5) The conformation of the MeS-C group remains as yet unpredictable, and was therefore approximated by choosing it coplanar with the double bond.

\section{RESULTS}

The starting positions of the four isomers 1a-d for the quantitative LIS-analysis are schematically drawn in Fig. 4.

Calculations were performed by evaluating the agreement factor between experimental and with the McConnell-Robertson equation calculated LIS, for all combinations of experimental LIS and atomic coordinates of the isomers. This affords 16 agreement factors, of which those resulting from correct combinations should show the lowest values. The results are given in Table 1.

From Table 1 it can be deduced that each set of experimental LIS of the four isomers affords the lowest agreement factors with their corresponding set of atomic coordinates (bold figures on the diagonal), as compared with the non-fitting combinations.

The values of the agreement factors for the correct combinations all occur in the range 0.03-0.08 which suggests that they can largely be explained by random experimental errors.

Only the agreement factors in the first and third row do not permit a discrimination between 1a, $1 \mathbf{b}$ and $1 \mathrm{c}$ and

Table 1. Agreement factors for all combinations of experimental LIS and atomic coordinates of isomers la-d

\begin{tabular}{|c|c|c|c|c|}
\hline & \multicolumn{4}{|c|}{ Atomic coordinates } \\
\hline & 1a & lb & 1c & Id \\
\hline la & 0.073 & 0.093 & 0.078 & 0.478 \\
\hline lb & 0.200 & 0.068 & 0.170 & 0.448 \\
\hline 马ु $1 c$ & 0.048 & 0.050 & 0.025 & 0.428 \\
\hline కี 1d & 0.352 & 0.341 & 0.271 & 0.078 \\
\hline
\end{tabular}

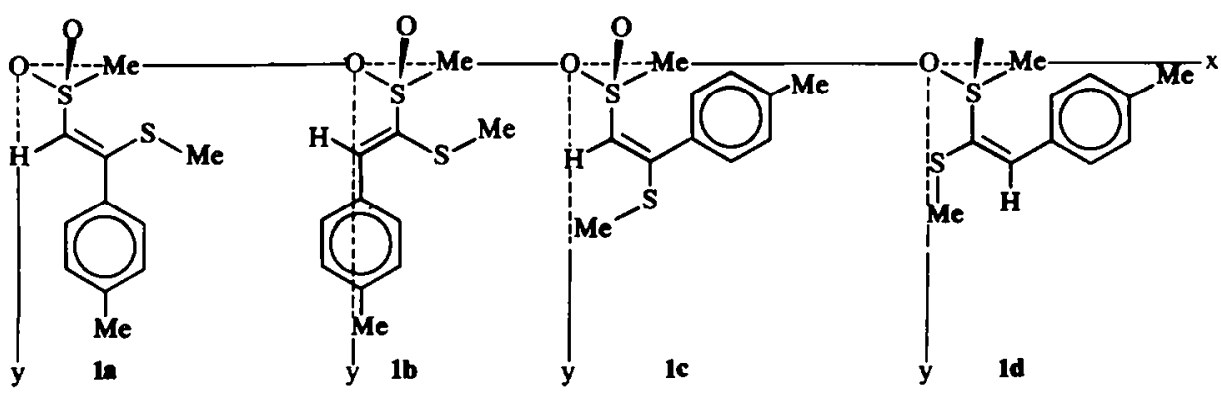

Fig. 4. Starting positions for LIS-analysis for the isomers la-d. 
between la and 1c respectively, on statistical grounds at the $5 \%$ level. ${ }^{15.16}$

Although the above results seem to indicate that the LIS of the isomers can directly be correlated with their structural features, the choice of the conformation around the $\mathrm{MeSO}_{2}-\mathrm{C}$ bond is still an uncertain factor, which needs further investigation. Therefore, in order to check whether this conformation has any physical meaning, the agreement factors for the isomers were calculated at different rotational positions of the molecule around this bond.

The starting positions were chosen essentially as depicted in Fig. 4, with the only difference that the double bond with substituents was coplanar with the methyl group attached to $-\mathrm{SO}_{2}$. The molecule was then rotated counter-clockwise around the $\mathrm{MeSO}_{2}-\mathrm{C}$ bond with

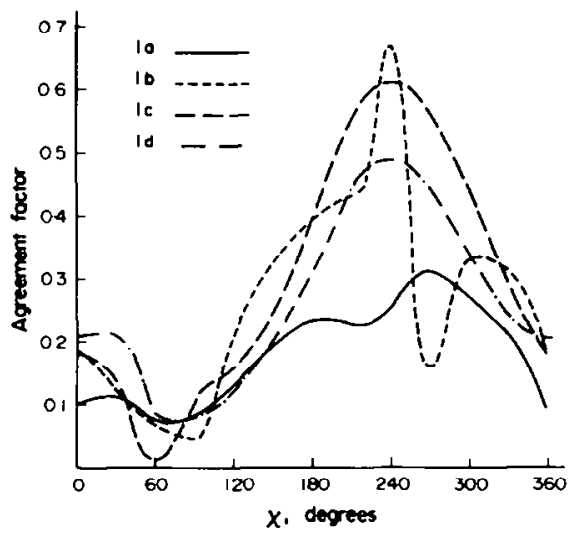

Fig. S. Dependency of agreement factor on rotation angle $x$ around $\mathrm{MeSO}_{2}-\mathrm{C}$ bond for isomers la-d. intervals of $30^{\circ}$. The dependency of the agreement factors on the rotation angles is shown in Fig. 5.

From the graphs in Fig. 5 it can be deduced that our prior assumption on the conformations of the isomers seems to be correct, since the minimum values for the A.F. are reached in the $60-90^{\circ}$ range. Moreover, the conformity of the minima in the graphs of Fig. 5 adds further evidence to the correctness of the structures of the isomers.

Dreiding models of the isomers indicate that the maxima of the graphs in Fig. 5 represent conformations which are unlikely or sterically even impossible. Also the effect of rotation around the $-\mathrm{SMe}$ bond upon the agreement factor was investigated. The results are shown in Fig. 6.

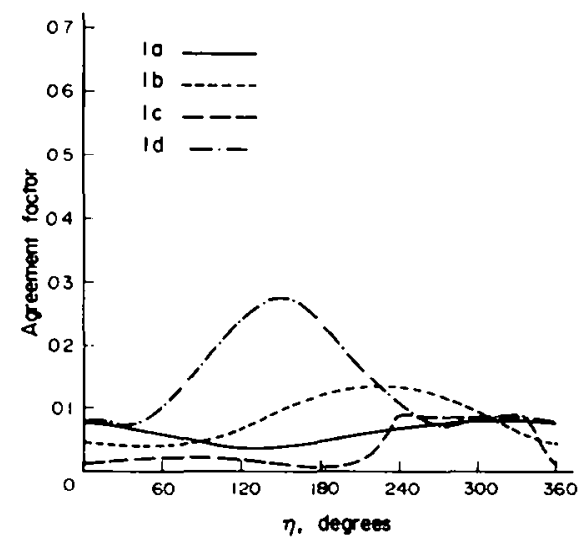

Fig. 6. Dependency of agreement factor on rotation angle $\eta$ around MeS-C bond for isomers la-d.

Table 2. LIS data for isomers $1 \mathrm{a}-\mathrm{d}^{a}$

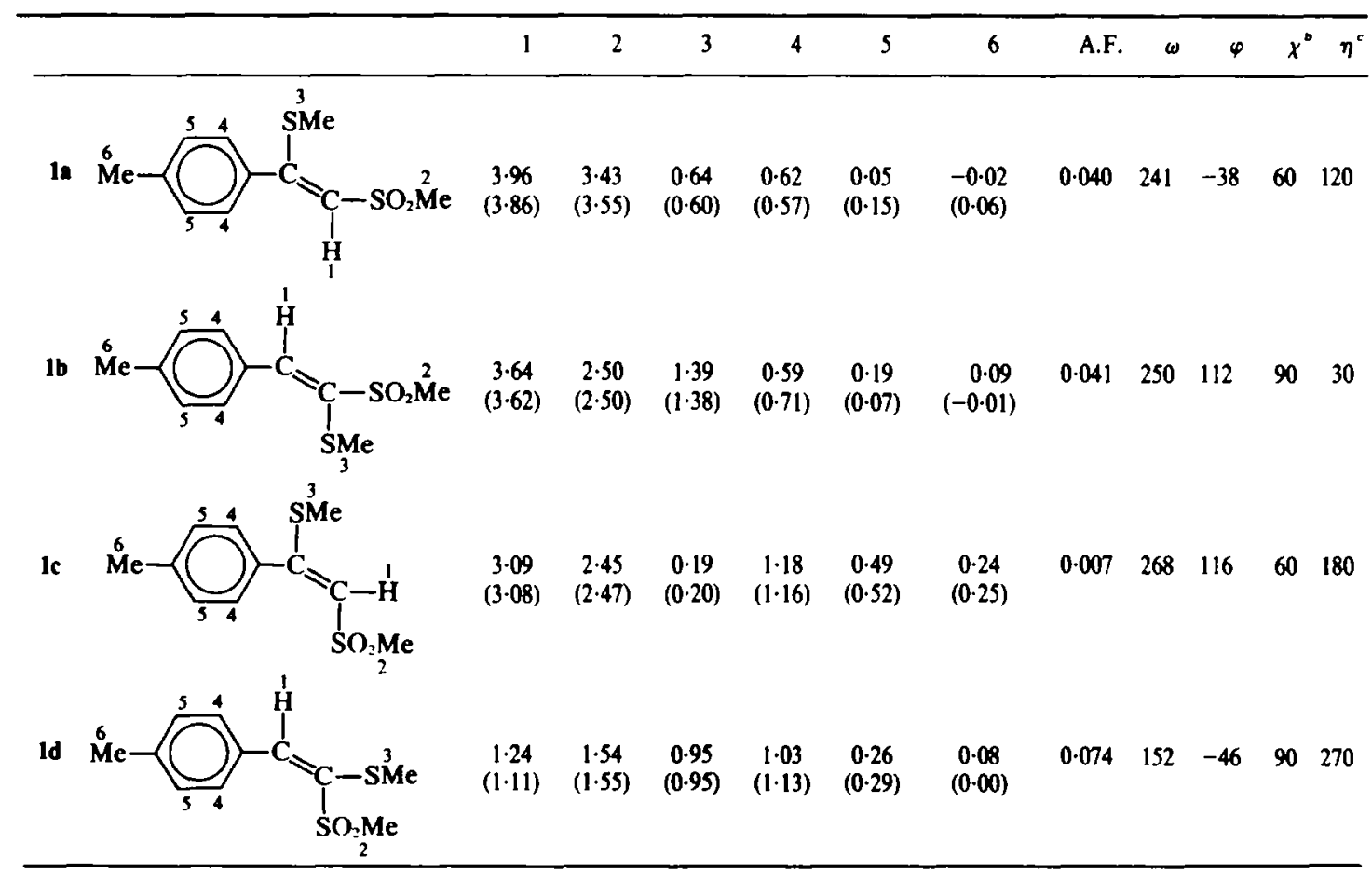

-Figures in columns 1-6 indicate experimental induced chemical shifts for Lanthanide/substrate ratio amounting to 0.5. Values in parentheses are calculated LIS.

Rotation angle around $\mathrm{MeSO}_{-}-\mathrm{C}$ bond.

'Rotation angle around MeS-C bond. 
Although the effects are less dramatic than those caused by rotation around the $\mathrm{MeSO}_{2}-\mathrm{C}$ bond, it is easily seen that the maxima in the graphs occur again at sterically unfavourable conformations.

The final results of the agreement between experimental and calculated induced chemical shifts are given in Table 2.

\section{CONCLUSIONS}

The foregoing results show that a LIS analysis is a valuable tool for discriminating between structurally closely related compounds. Although this had already been established for rigid compounds, this paper furnishes evidence for an extension to flexible systems, provided the proper assumptions with respect to the conformation of the flexible groups are chosen.

If on the other hand the molecular geometry of the compounds is known, a LIS may give useful information about the conformation of the molecules in solution, as was already pointed out in earlier reports. ${ }^{10,17}$.

\section{EXPERIMENTAI}

The compounds used in this study were prepared as described before. LIS measurements were performed at ambient temperature in $\mathrm{CDCl}_{3}$ using a Varian HA-100 NMR spectrometer. Spectra were recorded at Lanthanide/substrate molecular ratios in the range $0-1 \cdot 00$, increasing stepwise with $0 \cdot 125$. Aliquots of Lanthanide Shift Reagent were added from a stock soln in $\mathrm{CDCl}_{3}$ containing $0.83 \mathrm{M}$ of Eu(fod), using a $50 \mu$ syringe.

The experimental LIS at Lanthanide/substrate ratio 0.5 were obtained from a least squares fit of the data points. Calculations for the LIS simulation process were performed using a CDC, Cyber 73-26 computer system.
Acknowledgement -I wish to thank Mr. A. B. Verweij and Miss T. Volp for valuable technical assistance.

\section{RPFERTACRS}

'H. A. Selling, Tetrahedron. To be published.

${ }^{2}$ K. K. Andersen and J. J. Uebel, Tetrahedron Letters 5253 (1970),

${ }^{\prime} R$. R. Fraser and Y. Y. Wigfield, J. Chem. Soc. D, 1471 (1970).

${ }^{4}$ R. M. Wing. J. J. Uebel and K. K. Andersen, J. Am. Chem. Soc. 95, 6046 (1973)

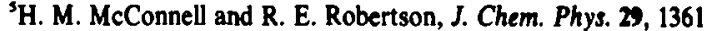
(1958).

'S. Farid, A. Ateya and M. Maggio, Chem. Comm. 1285 (1971).

${ }^{7}$ M. R. Willcott, III, R. E. Lenkinski and R. E. Davis, J. Am. Chem. Soc. 94, 1742 (1972).

'M. Ochiai, E. Mizuta, O. Aki, A. Morimoto and T. Okada, Tetrahedron Letters 3245 (1972)

'P. V. Demarco, B. J. Cerimele, R. W. Crane and A. L. Thakkar, Ibid. 3539 (1972)

${ }^{10} \mathrm{G}$. Montaudo, S. Caccamese, V. Librando and P. Maravigna, Tetrahedron 29, 3915 (1973).

"W. D. Horrocks, Jr. and J. P. Sipe, III, J. Am. Chem. Soc. 93, 6800 (1971)

${ }^{12} \mathrm{H}$. Huber, Tetrahedron Letters. 3559 (1972).

${ }^{13}$ W. H. Lawton and E. A. Sylvestre, Technometrics 13, 461 (1971).

${ }^{14}$ R. Hooke and T. A. Jeeves, J. Assoc. Computing Machinery 8, 212 (1961).

'W. C. Hamitton, Acta Cryst. 18, 502 (1965).

'R. E. Davis and M. R. Willcott, III, J. Am. Chem. Soc. 94, 1744 (1972).

1'J. D. Roberts, G. E. Hawkes, J. Husar, A. W. Roberts and D. W. Roberts, Tetrahedron 30, 1833 (1974)

I"G. E. Hawkes, D. Leibfritz, D. W. Roberts and J. D. Roberts, J. Am. Chem. Soc. 95, 1659 (1973). 